\title{
HYPOELLIPTIC VECTOR FIELDS AND CONTINUED FRACTIONS
}

\author{
STEPHEN J. GREENFIELD ${ }^{1}$
}

\begin{abstract}
We consider global analytic hypoellipticity of constant coefficient differential operators on the 2-torus, and prove that it is equivalent to a growth condition on the symbol. An example of a constant coefficient vector field which is globally analytic hypoelliptic but not globally hypoelliptic is constructed. Similar results are true on compact homogeneous spaces.
\end{abstract}

1. We discuss global analytic hypoellipticity for invariant differential operators on $T^{2}$, and contrast it with global hypoellipticity (the topic of [G]). In particular, we construct a "constant coefficient" vector field on $T^{2}$ which is globally analytic hypoelliptic but not globally hypoelliptic.

2. Let $T^{2}=\left\{\left(\exp i \theta_{1}, \exp i \theta_{2}\right) ; \theta_{j} \in \boldsymbol{R}\right\}$. If $L \in \mathfrak{D}^{\prime}\left(T^{2}\right)$ (a distribution on $\left.T^{2}\right)$ define $\hat{L}(n, m)=L\left(\exp \left(-i n \theta_{1}-i m \theta_{2}\right)\right)$. Suppose $P$ is an invariant differential operator: $P=\sum_{k, l=0}^{N} a_{k l} D_{1}^{k} D_{2}^{l}$, where $a_{k l} \in C$ and $D_{j}^{k}=$ $\left((1 / i) \partial / \partial \theta_{j}\right)^{k}$. Define $\hat{P}(n, m)=\sum_{k, l=0}^{N} a_{k l} n^{k} m^{l}$, for $n, m$ integers.

We say $P$ is globally hypoelliptic when:

(GH) If $g \in C^{\infty}\left(T^{2}\right)$, and $P f=g, f \in D^{\prime}\left(T^{2}\right)$, then $f \in C^{\infty}\left(T^{2}\right)$.

Let $A\left(T^{2}\right)$ denote the real analytic functions on $T^{2}$. We say $P$ is globally analytic hypoelliptic when:

(GAH) If $g \in A\left(T^{2}\right)$, and $P f=g, f \in D^{\prime}\left(T^{2}\right)$, then $f \in A\left(T^{2}\right)$.

The following theorem was proved in [G].

THEOREM 2.1. $P$ is $(\mathrm{GH})$ if and only if there are positive real numbers $L$ and $M$ so that:

(LM) $|\hat{P}(n, m)| \geqq L /\left(n^{2}+m^{2}\right)^{M}$, for $|n|,|m|$ sufficiently large.

An analogous characterization of (GAH) is given below.

THEOREM 2.2. $\quad P$ is (GAH) if and only if for any positive real number $K$ there is a positive integer $N_{K}$ so that:

$(\mathrm{KN})|\hat{P}(n, m)| \geqq \exp \left(-K\left(n^{2}+m^{2}\right)^{1 / 2}\right)$, for $|n|,|m|$ larger than $N_{K}$.

Received by the editors March 10, 1971.

AMS 1970 subject classifications. Primary 35H05, 42A92, 43A75.

Key words and phrases. Fourier coefficients, continued fractions, global hypoellipticity.

${ }^{1}$ Partially supported by NSF Grant number GP-20647.

()American Mathematical Society 1972 
Proof. We recall (see [K, p. 26]) that when $h \in \mathfrak{D}^{\prime}\left(T^{2}\right), h \in A\left(T^{2}\right)$ if and only if there exist positive constants $a$ and $b$ so that

$\left(^{*}\right)|\hat{h}(n, m)| \leqq b \exp \left(-a\left(n^{2}+m^{2}\right)^{1 / 2}\right)$.

Suppose $P f=g$, with $f$ and $g$ in $\mathcal{D}^{\prime}\left(T^{2}\right)$. Then $\hat{P}(n, m) \hat{f}(n, m)=\hat{g}(n, m)$. The sufficiency of $(\mathrm{KN})$ is demonstrated by applying $\left(^{*}\right)$ to $\hat{g}$ and using $(\mathrm{KN})$ to obtain $\left({ }^{*}\right)$ for $\hat{f}$. If $(\mathrm{KN})$ does not hold it is easy to create $g \in A\left(T^{2}\right)$ and $f \in \mathfrak{D}^{\prime}\left(T^{2}\right)-A\left(T^{2}\right)$ with $P f=g$.

Corollary 2.1. If $P$ is (GAH), then $P: A\left(T^{2}\right) \rightarrow A\left(T^{2}\right)$ is Fredholm of index 0.

Proof. $P$ is continuous. By $(\mathrm{KN}), \hat{P}(n, m) \neq 0$ when $|n|+|m|$ is sufficiently large. Kernel and cokernel are readily seen to have finite and equal dimension.

Corollary 2.2. If $P$ is $(\mathrm{GH})$, then $P$ is $(\mathrm{GAH})$.

Proof. (LM) implies (KN).

REMARK. The converse of Corollary 2.2 is not true, as we show in $\S 4$. We now recall some number theory in preparation.

3. We establish notation and recall theorems to be used in the remainder of this paper.

A finite (simple) continued fraction is a number $\alpha_{j}=\left[a_{1}, \cdots, a_{j}\right]$, where the $a_{k}$ 's are positive integers, and $\left[a_{1}, \cdots, a_{j}\right]$ is defined recursively by: $1 / a_{1}=\left[a_{1}\right],\left[a_{1}, \cdots, a_{j-1}+1 / a_{j}\right]=\left[a_{1}, \cdots, a_{j}\right]$. General references to continued fractions are $[\mathbf{H}]$ and $[\mathbf{S}]$.

THEOREM 3.1. Let $\alpha$ be a real number, with $0<\alpha<1$. Then there is a unique sequence of positive integers $\left\{a_{j}\right\}$ so that if $\alpha_{n}=\left[a_{1}, \cdots, a_{n}\right]$, then $\lim _{n \rightarrow \infty} \alpha_{n}=\alpha$.

If any such sequence is given, then $\lim _{n \rightarrow \infty} \alpha_{n}=\alpha$ always exists, with $0<\alpha<1$.

We define the infinite (simple) continued fraction $\alpha=\left[a_{1}, \cdots, a_{n}, \cdots\right]$ to be $\lim _{n \rightarrow \infty}\left[a_{1}, \cdots, a_{n}\right]$.

$\left[a_{1}, \cdots, a_{n}\right]=p_{n} / q_{n}$, where $p_{n}$, and $q_{n}$ are positive integers, and $\left(p_{n}, q_{n}\right)=1$. In what follows, $\alpha$ is assumed irrational.

THEOREM 3.2. $q_{n}=a_{n} q_{n-1}+q_{n-2}$. Thus $q_{n}$ is an increasing sequence of positive integers, and

$$
\frac{1}{\left(a_{n+1}+2\right) q_{n}^{2}}<\left|\alpha-\frac{p_{n}}{q_{n}}\right|<\frac{1}{a_{n+1} q_{n}^{2}} .
$$


The key property of continued fractions to be used is that of "best approximation", as given in the following result:

THEOREM 3.3. Suppose $p$ and $q$ are positive integers, and $0<q \leqq q_{n}$. Then $\left|p_{n}-q_{n} \alpha\right|<|p-q \alpha|$.

We can now construct an interesting example.

4. Let $\alpha=\left[a_{1}, \cdots, a_{n}, \cdots\right]$, where $a_{n}=10^{n !}$.

THEOREM 4.1. The vector field $V=D_{1}-\alpha D_{2}$ is (GAH) but not (GH).

Proof. $\alpha$ is a Liouville number [H, p. 162]. Thus [G] shows that $V$ is not (GH). We prove that $V$ is (GAH) by showing (A) below, which clearly implies (KN) for $V$.

(A) Given $K>0$, there is $Q$ so that for $q \geqq Q,|p-\alpha q| \geqq \exp (-K q)$, $p, q$ positive integers.

(Note that $\hat{V}(p, q)=p-\alpha q$.)

Using the best approximation property, we need only prove an apparently weaker statement:

(B) Given $K>0$, there is an integer $N$ so that $\left|p_{n}-\alpha q_{n}\right| \geqq$ $\exp \left(-K q_{n-1}\right)$ for $n \geqq N$.

(Because (B) gives (A) when $q \geqq q_{N-1}$.)

By Theorem 3.2,

$$
\begin{gathered}
\frac{1}{\left(a_{n+1}+2\right) q_{n}} \leqq\left|q_{n} \alpha-p_{n}\right| \\
a_{n+1}=10^{(n+1) !}=\left(10^{n !}\right)^{n+1}=\left(a_{n}\right)^{n+1}
\end{gathered}
$$

so we certainly can estimate:

But $q_{n} \geqq a_{n}$, so that

$$
\frac{1}{\left(a_{n}\right)^{n+2} q_{n}} \leqq\left|q_{n} \alpha-p_{n}\right| .
$$

$$
\frac{1}{\left(q_{n}\right)^{n+3}} \leqq\left|q_{n} \alpha-p_{n}\right| .
$$

(It is interesting to contrast our goal, (B), with the statement of Hardy and Wright establishing that $\alpha$ is a Liouville number: $\left|q_{n} \alpha-p_{n}\right| \leqq$ $1 /\left(q_{n}\right)^{N / 2+1}$, for $n \geqq N$.)

So (B) will be proven if we show that

(C) $\left(q_{n}\right)^{n+3} \leqq \exp \left(K q_{n-1}\right), n$ large.

But (C) means: $(n+3) \log q_{n} \leqq K q_{n-1}$. Or $n \log q_{n} \leqq K^{\prime} q_{n-1}, K^{\prime}=K / 2$, $n$ large enough. But $q_{n}=a_{n} q_{n-1}+q_{n-2} \leqq\left(a_{n}+1\right) q_{n-1} \leqq\left(a_{n}\right)^{2} q_{n-11}$ $\log q_{n} \leqq 2 \log a_{n}+\log q_{n-1}$. And (C) is true if $2 n(n !) \log 10 \leqq K^{\prime \prime} q_{n-\text {. }}$. and $n \log q_{n-1} \leqq K^{\prime \prime} q_{n-1}\left(K^{\prime \prime}=K^{\prime} / 2\right)$ for $n$ large enough. This can be done 
by elementary manipulations, since $q_{n} \geqq a_{n}$, and $a_{n}=10^{n !}$. (Roughly, (C) is true since $10^{n !}$ is rather large. Conversations with $\mathrm{K}$. Johnson and N. Wallach were quite useful in assembling this proof.)

5. There are (many) vector fields which are not hypoelliptic. Here is one example:

THEOREM 5.1. There is an irrational $\alpha, \alpha=\left[a_{1}, \cdots, a_{n}, \cdots\right]$, so that the vector field $V=D_{1}-\alpha D_{2}$ is neither (GAH) nor (GH).

PRoof. $V$ is not hypoelliptic if there is $K>0$ so that

$$
\left|q_{n} \alpha-p_{n}\right|<\frac{1}{a_{n+1} q_{n}} \exp \left(-K q_{n}\right) .
$$

(For surely $1 \leqq p_{n} \leqq q_{n}$, so that $\exp \left(-K q_{n}\right) \leqq \exp \left(-K^{\prime}\left(q_{n}^{2}+p_{n}^{2}\right)^{1 / 2}\right)$, with some appropriate $K^{\prime}$.)

By Theorem 3.2, $q_{n}$ is computable knowing only $a_{1}, \cdots, a_{n}$. Let $K>0$ be given. Define $\alpha: a_{1}=1$, and $a_{n+1}$ is any integer larger than $\exp \left(K q_{n}\right) / q_{n} . \alpha$ is insured to exist by Theorem 3.1, and of course it satisfies (D).

REMARK 5.2. The reasoning above arose in a conversation with Professor W. Schmidt. Similarly one can show the existence of $\alpha$ 's so that $|q \alpha-p| \leqq G\left(\left(q^{2}+p^{2}\right)^{1 / 2}\right)$ for an infinite number of integral pairs $(p, q)$, and any decreasing function $G$. From this perspective, the vector field of Theorem 4.1 was (GAH) because the rate of growth of $a_{n}$ was not fast enough.

6. The results of $\S 2$ are, of course, true in a wider context. In particular, we cite invariant differential operators on vector bundles over compact homogeneous spaces (see [W]).

\section{BIBLIOGRAPHY}

G. Stephen J. Greenfield and Nolan R. Wallach, Global hypoellipticity and Liouville numbers, Proc. Amer. Math. Soc. 31 (1972), 112-114.

H. G. H. Hardy and E. M. Wright, An introduction to the theory of numbers, Oxford Univ. Press, London, 1954. MR 16, 673.

K. Y. Katznelson, An introduction to harmonic analysis, Wiley, New York, 1968. MR 40 \#1734.

S. H. M. Stark, An introduction to number theory, Markham, Chicago, Ill., 1970. MR 40 \#7186.

W. Nolan R. Wallach, Differential operators on homogeneous spaces, Marcel Dekker, New York (to appear).

Department of Mathematics, Rutgers University, New Brunswick, New JERSEY 08903 